\title{
Species richness of anthophilous butterflies of an Atlantic Forest fragment in Southeastern Brazil
}

\author{
Kelen Coelho Cruz ${ }^{1}$, Sileimar Maria Lelis ${ }^{1}$, Mariana Aparecida Silva Godinho ${ }^{1}$, Rúbia Santos Fonseca ${ }^{2}$, \\ Paulo Sérgio Fiúza Ferreira ${ }^{3}$, Milene Faria Vieira ${ }^{4}$
}

\begin{abstract}
The objectives of this study were to identify anthophilous butterflies on psychophilous flowers of four Asteraceae species in an Atlantic Forest fragment in Viçosa, Minas Gerais State, Southeastern Brazil, and to determine whether there are species in common with other lepidopteran inventories of the Southeastern and Midwestern regions of Brazil. It is the first inventory of anthophilous butterflies of a semideciduous forest fragment in Zona da Mata, State of Minas Gerais. A total of 108 species were recorded, representing the fourth largest lepidopteran survey in this State. The results demonstrated that Asteraceae species may be important tools for monitoring anthophilous butterflies. The similarity with other inventories ranged from 1 to $92.55 \%$. Fifteen species were reported for the first time in the State of Minas Gerais, and among them, Melanis alena and Thisbe irenea were observed in this study only.
\end{abstract}

Key words: Asteraceae, Brazil, psychophily, Seasonal Semideciduous Forest.

\section{RESUMO}

\section{Riqueza de espécies de borboletas antófilas de um fragmento de Mata Atlântica no Sudeste do Brasil}

Os objetivos do presente trabalho foram identificar borboletas antófilas em flores psicófilas de quatro espécies de Asteraceae em um fragmento de Floresta Atlântica, no município de Viçosa, Minas Gerais, Sudeste brasileiro, e determinar se existem espécies de borboletas em comum com outros levantamentos de lepidópteros do Sudeste e do CentroOeste do Brasil. É o primeiro inventário de borboletas antófilas de um fragmento de Floresta Estacional Semidecidual na Zona da Mata de Minas Gerais. Foram registradas 108 espécies, representando o quarto maior levantamento de lepidópteros de Minas Gerais. Esses resultados demonstraram que espécies de Asteraceae podem ser importantes ferramentas para o monitoramento de borboletas antófilas. A similaridade com outros inventários variou de 1 a 92,55\%. Quinze espécies foram reportadas pela primeira vez em Minas Gerais e, entre elas, Melanis alena e Thisbe irenea foram observadas somente nesse estudo.

Palavras-chave: Asteraceae, Brasil, psicofilia, Floresta Estacional Semidecidual.

\footnotetext{
Received for publication on January $24^{\text {th }}, 2012$ and approved on August $30^{\text {th }}, 2012$

Biologist, Master of Science. Departamento de Biologia Vegetal, Universidade Federal de Viçosa, Campus Viçosa, Avenida Peter Henry Rolfs, s/n, 36570-000, Viçosa, Minas Gerais, Brasil. kelencruz@yahoo.com.br; sileimarbot@hotmail.com; marianagodinhobot@gmail.com

${ }^{2}$ Biologist, Master of Science. Doctoral student at Programa de Pós-Graduação em Botânica, Departamento de Biologia Vegetal, Universidade Federal de Viçosa, Campus Viçosa, Avenida Peter Henry Rolfs, s/n, 36570-000, Viçosa, Minas Gerais, Brasil. rubiafonsecabot@gmail.com

${ }^{3}$ Biologist, PhD. Departamento de Biologia Animal, Universidade Federal de Viçosa, Campus Viçosa, Avenida Peter Henry Rolfs, s/n, 36570-000, Viçosa, Minas Gerais, Brasil. pfiuza@ufv.br

${ }^{4}$ Agronomist Engineer, Doctor of Science. Departamento de Biologia Vegetal, Universidade Federal de Viçosa, Campus Viçosa, Avenida Peter Henry Rolfs, s/n, 36570-000, Viçosa, Minas Gerais, Brasil. mfvieira@ufv.br (corresponding author).
} 


\section{INTRODUCTION}

Atlantic Forest is one of the most threatened and destroyed biomes of the planet. Today, the remaining forests cover about $100,000 \mathrm{~km}^{2}$, corresponding to $1 \%$ of the Brazilian territory (Fundação SOS Mata Atlântica, 2009). In the State of Minas Gerais, Southeastern Brazil, the Atlantic Forest covers about $10.33 \%$ of the territory (IEF, 2010), including the region known as Zona da Mata of Minas Gerais.

The municipality of Viçosa is located in the Zona da Mata and originally its vegetation consisted of continuous forests inserted in Atlantic Forest domains, which is classified as Montane Semideciduous Forest (Veloso et $a l ., 1991)$. The original vegetation was replaced by coffee plantations for some time, and today the vegetation is predominantly pastureland covered with molasses grass (Mellinis minutiflora Beauv.) and secondary forests in different successional stages.

The forest reserve Mata do Paraíso, site of the present study, is the largest forest fragment in Viçosa. In the 19th century, after deforestation, it was partly replaced by coffee plantations and pastures. Nowadays, the forest in different succession stages covers an area of 194.36 ha (Pinto et al., 2007) and represents a vegetation island.

Added to the drastic reduction of the Viçosa's flora, only two studies involving the fauna of lepidopterous were carried out, both in Mata do Paraíso: inventories of the species Sphingidae and Arctiidae, but without relating the species to their host plants (Ferreira et al., 1986; 1995). These surveys reported 21 new occurrences of Arctiidae in Minas Gerais. Recently, in studies performed by Godinho (2007), Lelis (2008), Cruz (2009) and Fonseca (2009), also carried out in Mata do Paraíso, four species of Asteraceae were described as pollinated by butterflies (psychophily) and the high number of species of these insects visiting their flowers motivated this study.

According to Mani \& Saravanan (1999), about $75 \%$ of the Asteraceae species are psychophilous. Their floral characteristics, such as brightly colors, no perceptible odor, narrow tubular corolla and concealed nectar at the corolla base are conforming to the butterfly pollination syndrome. Besides, the aggregated arrangement of flowers on the inflorescence (capitulum), forming a landing platform, is another important attribute.

The objectives of this study were to identify anthophilous butterflies (visiting flowers and feeding on nectar), visitors of four Asteraceae species in the forest reserve Mata do Paraíso, and to compare the recorded lepidopteran species, especially butterflies, with other inventories of the Southeast and Midwest regions of Brazil.

\section{MATERIALS AND METHODS}

\section{Study site}

The climate in the region of Viçosa $\left(20^{\circ} 48^{\prime} 07^{\prime \prime S}\right.$ and $42^{\circ} 51^{\prime} 31^{\prime \prime} \mathrm{W}, 680 \mathrm{~m}$ ) is characterized by a cold and dry season (from April to September), with monthly averages of $17^{\circ} \mathrm{C}$ and $32 \mathrm{~mm}$ precipitation, followed by a hot and rainy season (from October to March), with monthly averages of $21^{\circ} \mathrm{C}$ and $171 \mathrm{~mm}$ precipitation (Departamento Nacional de Meteorologia, 1992).

\section{Data collection and analysis}

Fieldwork was carried out in 2005, 2006, 2007 and 2008. Butterflies were collected from psychophilous flowers of Asteraceae species (Figure 1): Adenostemma brasilianum (Pers.) Cass., Bidens segetum Mart. ex Colla, Eupatorium intermedium DC. and Tilesia baccata (L.) Pruski. All plants are commonly found along forest edges.

Collections from $A$. brasilianum were performed in March-April 2005 and 2006 (Godinho, 2007), from $B$. segetum in April-May 2007 and 2008 (Lelis, 2008); from $E$. intermedium in February-March 2007 and March 2008 (Cruz, 2009), and from T. baccata in January 2008 (Fonseca, 2009). Butterflies were surveyed from 7:00-17:00, with a sampling effort of about 100 hours.

Specimens were killed by pinching the thorax, and then mounted and deposited at the Entomological Museum (Museu Regional de Entomologia), Departmento de Biologia Animal, Universidade Federal de Viçosa (UFV). Species were identified with specialists' assistance.

The species of this study were compared with those recorded in inventories of Minas Gerais State and the most representative inventories of São Paulo, Espírito Santo and Distrito Federal State, conducted in areas of Atlantic Forest, Cerrado and urban vegetation.

\section{RESULTS}

\section{Collected anthophilous butterflies}

A total of 108 anthophilous butterfly species were recorded on the flowers of four asteracean species in Mata do Paraíso, belonging to 70 genera, 16 subfamilies and five families (Table 1). Nymphalidae (48 species) and Hesperiidae (36 species) together represented $78 \%$ of the collected species. Among the subfamilies of Nymphalidae, Ithomiinae was represented by the largest number (23 species). For Hesperiidae, the subfamily Pyrginae was the most representative (19 species). Papilionidae, Pieridae and Riodinidae together accounted for about $22 \%$ of the surveyed species. 


\section{Comparison with other lepidopteran inventories}

The 108 species recorded in this study, along with the 125 species of Sphingidae and Arctiidae identified in other surveys in Mata do Paraíso (MP), represent the fourth largest inventory of Lepidoptera in Minas Gerais, totaling 233 species (Table 2). Compared to other surveys in the same State (Table 2), this number of records is close to the inventory in the region of Belo Horizonte (269 species), in areas of semideciduous forest, Cerrado, rocky fields and urban areas; and in Uberlândia (251 species), in similar areas. On the other hand, the number of species detected in Mata do Paraíso is about 2.5-fold lower than in Poços de Caldas, where the most comprehensive inventory of Minas Gerais (572 species) in Atlantic Forest was carried out.

The similarity of the anthophilous Lepidoptera fauna of Mata do Paraíso with the other sites ranged from 1 to $92.55 \%$ (Table 3). Over $90 \%$ of the 94 identified species were also found in São Paulo State (SP). The inventory of this State, carried out in the same vegetation as Mata do Paraíso, is the most representative of all surveys involving Atlantic Forest (652 species) (Table 2).
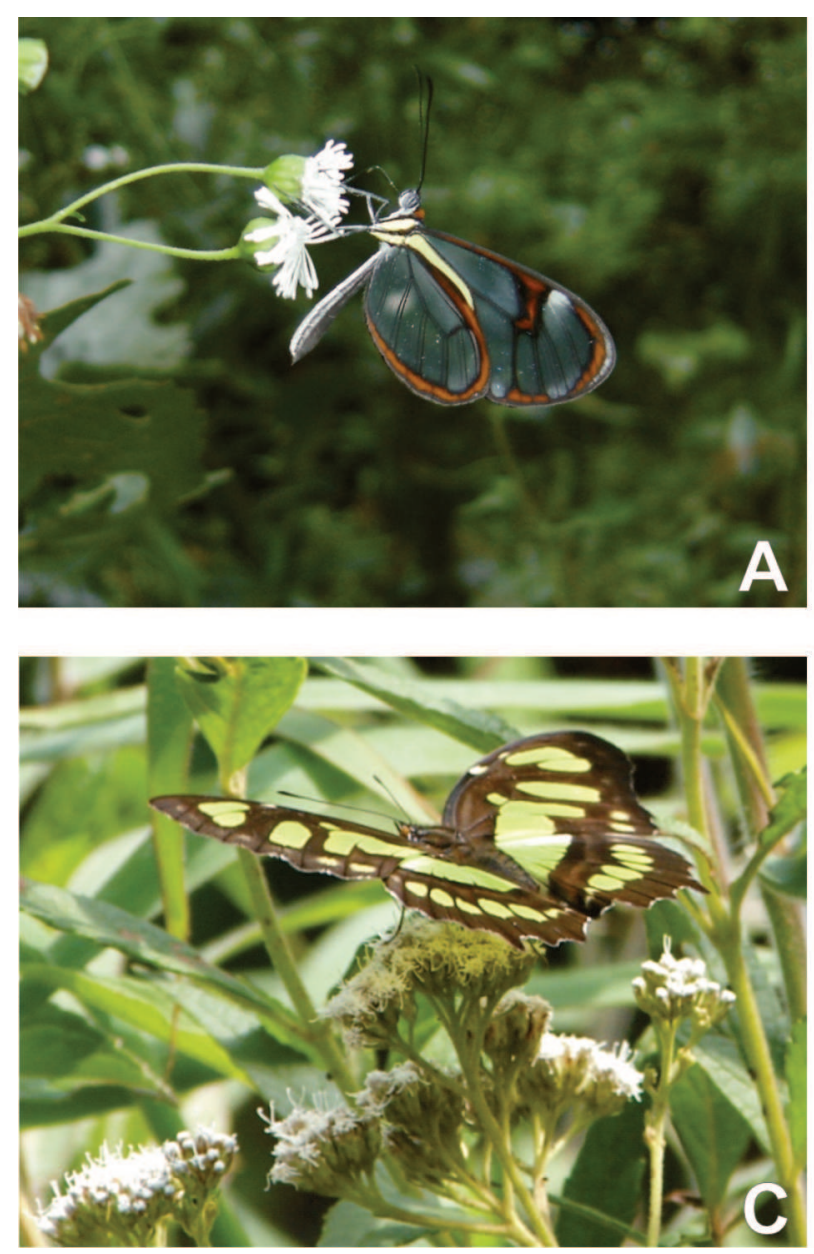

Comparing the survey of Mata do Paraíso with two others conducted in Atlantic Forest (in Santa Teresa, ES) and in Cerrado (in Brasília, DF), a distinct biome (Table 2), a high similarity was found with respectively, $78.72 \%$ and $70.21 \%$ butterfly species in common (Table 3 ).

In the State of Minas Gerais, the highest degree of similarity with the inventory of Mata do Paraíso was observed with Poços de Caldas (PC) and Belo Horizonte $\left(\mathrm{BH}_{1}\right), 63.82$ and $61.70 \%$, respectively (Table 3). Among the inventories conducted in Minas Gerais, these two contained the most representative number of species (Table 2). Besides, $69.56 \%$ of the Ithomiinae species found in Mata do Paraíso were also reported in the study carried out in Juiz de Fora (JF) (Table 2).

When comparing our results with those of other inventories, we found that 15 species were first reported in Minas Gerais State (Table 1). Among the new records, Melanis alena (Hewitson, 1870) and Thisbe irenea (Stoll, 1780), both belonging to Riodinidae, occurred only in Mata do Paraíso.
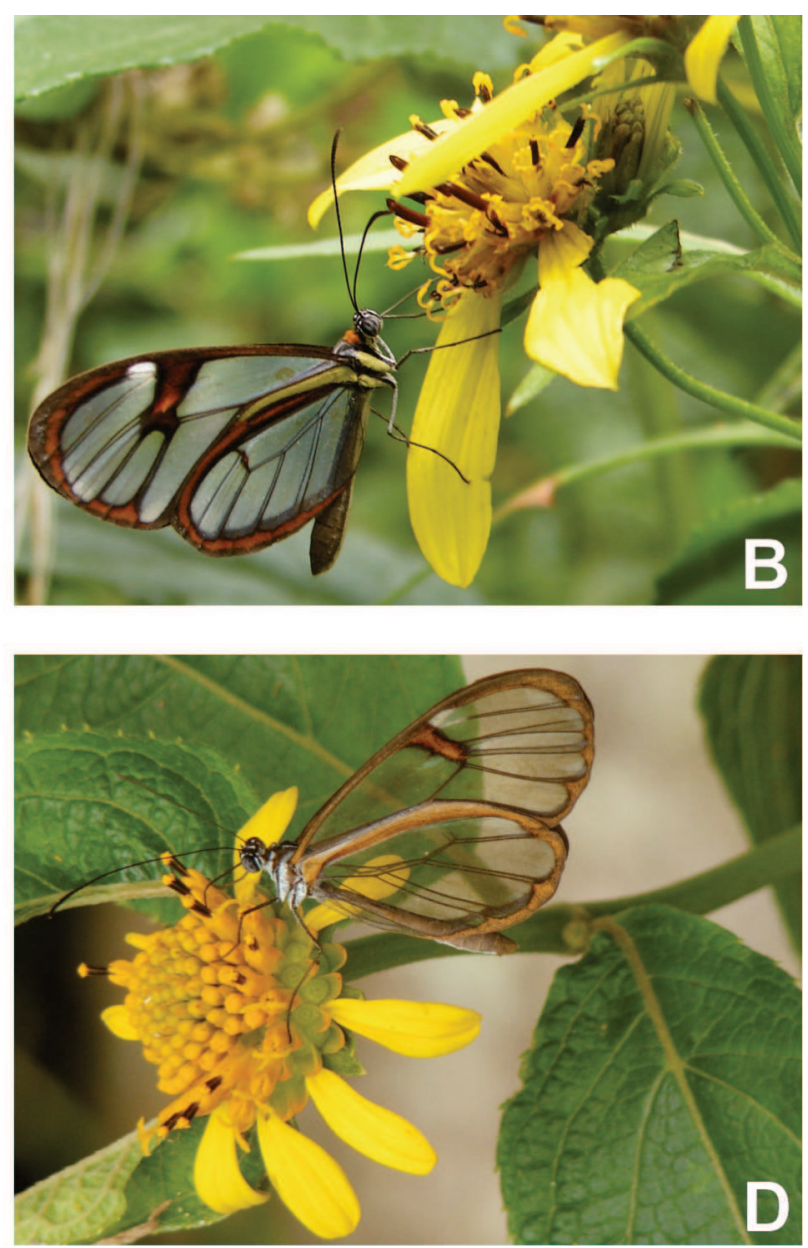

Figure 1. Psychophilous flowers of four Asteraceae species: A - Adenostemma brasilianum; B - Bidens segetum; C - Eupatorium intermedium; D - Tilesia baccata. In A, B and D the flowers are visited by species of Nymphalidae: Ithomiinae; in C by Siproeta stelenes (Nymphalidae: Nymphalinae). 
Table 1. Anthophilous butterflies (Lepidoptera: Rhopalocera) in Seasonal Semideciduous Forest in Viçosa, Minas Gerais State, Southeastern Brazil. The list also includes butterfly inventories from other collection sites in the Southeast Region (Espírito Santo, Minas Gerais and São Paulo) and Midwest Region (Distrito Federal) of Brazil. The letters after each butterfly species indicates its host plant: $\mathrm{a}=$ Adenostemma brasilianum $; \mathrm{b}=$ Bidens segetum $; \mathrm{e}=$ Eupatorium intermedium $; \mathrm{t}=$ Tilesia baccata

\begin{tabular}{|c|c|c|}
\hline Family/ Subfamily & Species & Collection sites $^{1}$ \\
\hline Hesperiidae/ Hesperiinae & $\begin{array}{l}\text { Eutychide physcella }(\text { Hewitson, 1866) } \\
\text { Moeris striga }(\text { Geyer, 1832) } \\
\text { Remella remus }(\text { Fabricius, 1798) } \\
\text { Nyctelius nyctelius }\left(\text { Latreille, }[1824]^{\mathrm{e}}\right. \\
\text { Pompeius pompeius }\left(\text { Latreille, [1824]) }{ }^{\mathrm{e}}\right. \\
\text { Vehilius stictomenes }(\text { Butler, 1877) } \\
\text { Morpho-species } 1^{\mathrm{e}, \mathrm{t}} \\
\text { Morpho-species } 2^{\mathrm{e}} \\
\text { Morpho-species } 3^{\mathrm{e}} \\
\text { Morpho-species } 4^{\mathrm{e}}\end{array}$ & $\begin{array}{l}\mathrm{PC}, \mathrm{ES}, \mathrm{SP} \\
\mathrm{PC}, \mathrm{ES}, \mathrm{SP}, \mathrm{DF} \\
\mathrm{BH}_{1}, \mathrm{PC}, \mathrm{SP} \\
\mathrm{PC}, \mathrm{UB}_{2,} \mathrm{ES}, \mathrm{SP}, \mathrm{DF} \\
\mathrm{PC}, \mathrm{UB}_{2}, \mathrm{ES}, \mathrm{SP}, \mathrm{DF} \\
\mathrm{BH}_{1,}, \mathrm{PC}, \mathrm{UB}_{2,}, \mathrm{ES}, \mathrm{SP}, \mathrm{DF} \\
- \\
- \\
-\end{array}$ \\
\hline Hesperiidae/ Pyrginae & 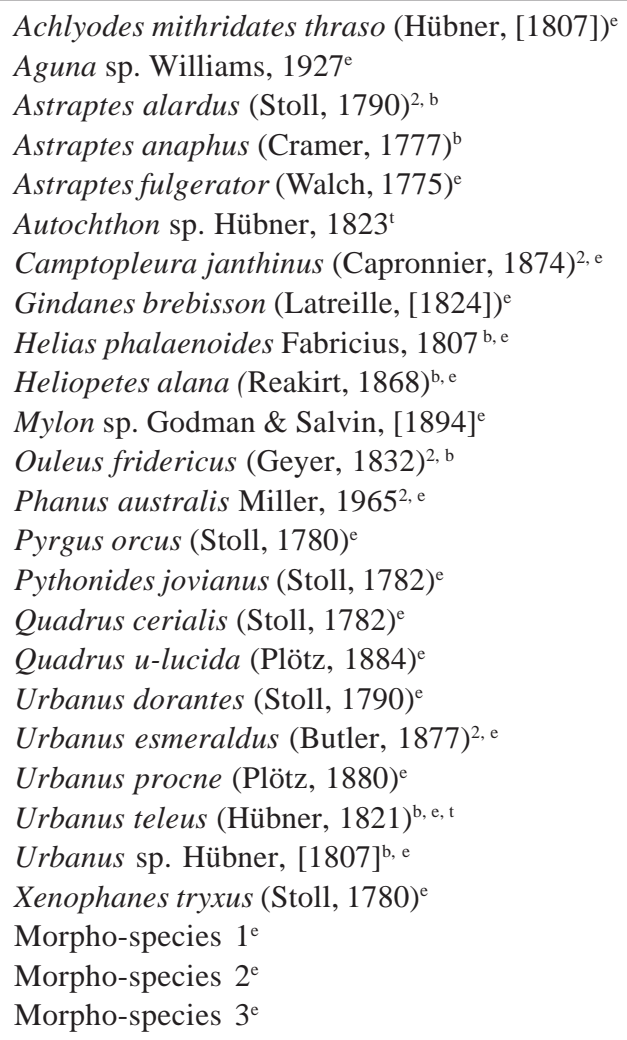 & 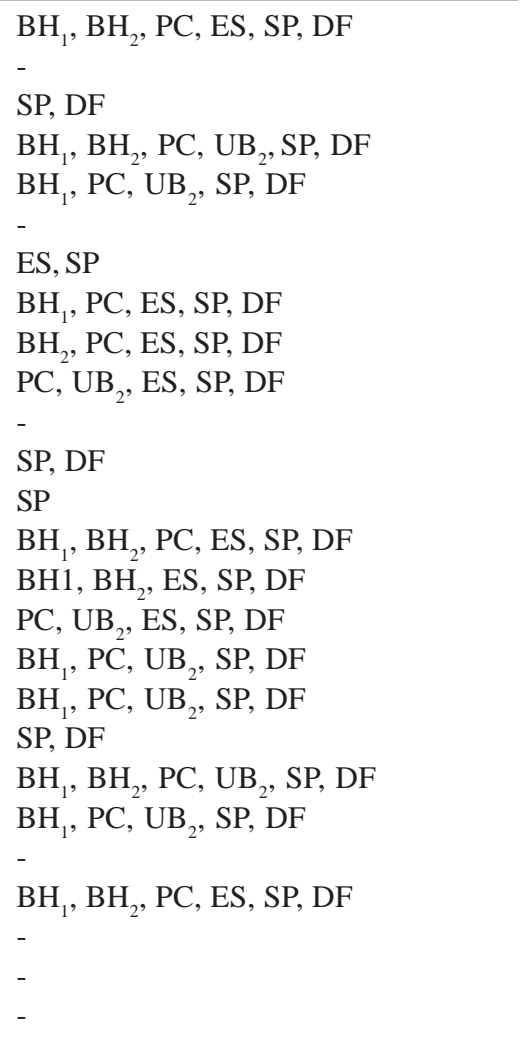 \\
\hline Nymphalidae/ Acraeinae & $\begin{array}{l}\text { Actinote carycina Jordan, } 1913^{\mathrm{e}} \\
\text { Actinote discrepans } \mathrm{D}^{\prime} \text { Almeida, } 1958^{2, \mathrm{~b}} \\
\text { Actinote pellenea Hübner }[1821]^{\mathrm{b}, \mathrm{e}} \\
\text { Actinote thalia pyrrha }(\text { Fabricius, } 1775)^{\mathrm{b}}\end{array}$ & $\begin{array}{l}\mathrm{PC}, \mathrm{UB}_{2}, \mathrm{ES}, \mathrm{SP}, \mathrm{DF} \\
\mathrm{SP}, \mathrm{DF} \\
\mathrm{BH}_{1}, \mathrm{BH}_{2}, \mathrm{UB}_{2}, \mathrm{ES}, \mathrm{SP}, \mathrm{DF} \\
\mathrm{BH}_{1}, \mathrm{BH}_{2}, \mathrm{PC}, \mathrm{ES}, \mathrm{SP}\end{array}$ \\
\hline Nymphalidae/ Biblidinae & $\begin{array}{l}\text { Biblis hyperia nectanabis (Fruhstorfer, 1909) } \\
\text { Temenis laothoe meridionalis Ebert, } 1965^{\mathrm{b}, \mathrm{e}}\end{array}$ & $\begin{array}{l}\mathrm{BH}_{1}, \mathrm{BH}_{2}, \mathrm{MG}_{1}, \mathrm{PC}, \mathrm{UB}_{2}, \mathrm{ES}, \mathrm{SP} \\
\mathrm{BH} 1, \mathrm{ES}, \mathrm{MG}_{1}, \mathrm{PC}, \mathrm{UB}_{1}, \mathrm{UB}_{2}, \mathrm{SP}, \mathrm{DF}\end{array}$ \\
\hline Nymphalidae/ Cyrestinae & Marpesia chiron (Fabricius, 1775$)^{\mathrm{e}}$ & $\mathrm{BH}_{1}, \mathrm{PC}, \mathrm{ES}, \mathrm{SP}, \mathrm{DF}$ \\
\hline Nymphalidae/ Danainae & Lycorea halia discreta (Haensch, 1909) & $\mathrm{BH}_{2}, \mathrm{ES}, \mathrm{SP}, \mathrm{DF}$ \\
\hline Nymphalidae/Heliconiinae & $\begin{array}{l}\text { Agraulis vanillae (Linnaeus, 1758) } \\
\text { Dryas iulia }(\text { Fabricius, 1775) } \\
\text { Eueides aliphera }(\text { Godart, 1819) } \\
\text { Eueides isabella }(\text { Stoll, } 1781)^{\mathrm{e}} \\
\text { Eueides pavana Ménétriés, } 1857^{\mathrm{e}} \\
\text { Heliconius erato phyllis }(\text { Fabricius, } 1775)^{\mathrm{b}, \mathrm{e}, \mathrm{t}} \\
\text { Heliconius ethilla } \text { Godart, } 1819^{\mathrm{b}, \mathrm{e}, \mathrm{t}} \\
\text { Heliconius sara }(\text { Fabricius, 1793) } \\
\text { Philaethria wernickei }(\text { Röber, 1906) }\end{array}$ & $\begin{array}{l}\mathrm{BH}_{1}, \mathrm{BH}_{2}, \mathrm{PC}, \mathrm{UB}_{2}, \mathrm{ES}, \mathrm{SP}, \mathrm{DF} \\
\mathrm{BH}_{1}, \mathrm{BH}_{2}, \mathrm{PC}, \mathrm{UB}_{2}, \mathrm{ES}, \mathrm{SP}, \mathrm{DF} \\
\mathrm{BH}_{1}, \mathrm{PC}, \mathrm{ES}, \mathrm{SP}, \mathrm{DF} \\
\mathrm{BH}_{1}, \mathrm{UB}, \mathrm{ES}, \mathrm{SP}, \mathrm{DF} \\
\mathrm{BH}_{1}, \mathrm{ES}, \mathrm{SP} \\
\mathrm{BH}_{1}, \mathrm{BH}_{2}, \mathrm{PC}, \mathrm{UB}_{2}, \mathrm{ES}, \mathrm{SP}, \mathrm{DF} \\
\mathrm{BH}_{1}, \mathrm{PC}, \mathrm{UB}_{2}, \mathrm{ES}, \mathrm{SP}, \mathrm{DF} \\
\mathrm{BH}_{1}, \mathrm{ES}, \mathrm{SP}, \mathrm{DF} \\
\mathrm{PC}, \mathrm{ES}, \mathrm{SP},\end{array}$ \\
\hline
\end{tabular}


Continuação tabela 1

Nymphalidae/ Ithomiinae

Aeria olena Weymer, $1875^{\text {a }}$

Brevioleria aelia plisthenes (d'Almeida, 1958)

Dircenna dero rhoeo Felder \& Felder, $1860^{\mathrm{a}, \mathrm{e}}$

Episcada carcinia Schaus, 1902 ${ }^{\mathrm{a}, \mathrm{b}, \mathrm{e}}$

Episcada clausina (Hewitson, 1876) 2, a, b, e

Episcada hymenaea (Prittwitz, 1865) ${ }^{\mathrm{a}}$

Episcada philoclea (Hewitson, [1855])2, b

Epityches eupompe (Geyer, [1832]) ${ }^{\mathrm{b}, \mathrm{e}}$

Hypoleria adasa (Hewitson, [1855]) ${ }^{\mathrm{e}}$

Hypoleria lavinia (Hewitson, [1855])2, e

Hypothyris euclea laphria (Doubleday, 1847)

Hypothyris ninonia (Hübner, [1806]) a, b, e

Ithomia agnosia Hewitson, [1855] a, b, e, t

Ithomia drymo Hübner, $1816^{\mathrm{b}, \mathrm{e}}$

Ithomia lichyi d'Almeida, 1939ª

Mechanitis lysimnia (Fabricius, 1793) a, b, e $^{\text {a }}$

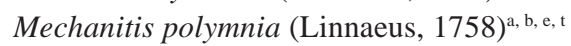

Oleria aquata (Weymer, 1875) ${ }^{\mathrm{a}}$

Placidina euryanassa (Felder \& Felder, 1860) ${ }^{\mathrm{a}}$

Pseudoscada erruca (Hewitson, 1855)

Pteronymia sylvo (Geyer, 1832) a, $^{\mathrm{a}}$

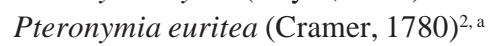

$\mathrm{BH}_{1}, \mathrm{BH}_{2}, \mathrm{JF}, \mathrm{PC}, \mathrm{UB}_{2}, \mathrm{ES}, \mathrm{SP}, \mathrm{DF}$

$\mathrm{BH}_{1}, \mathrm{DF}, \mathrm{UB}_{2}, \mathrm{SP}$

$\mathrm{BH}_{1}, \mathrm{JF}, \mathrm{PC}, \mathrm{UB}_{2}, \mathrm{ES}, \mathrm{SP}, \mathrm{DF}$

$\mathrm{BH}_{1}$, JF, PC, ES, SP

ES, SP

JF, PC, UB, ES, SP

SP

JF, PC, ES, SP

JF, ES, SP

DF

$\mathrm{BH}_{1}, \mathrm{BH}_{2}, \mathrm{ES}, \mathrm{SP}, \mathrm{DF}$

$\mathrm{JF}, \mathrm{UB}_{2}, \mathrm{ES}, \mathrm{SP}, \mathrm{DF}$

$\mathrm{BH}_{1}, \mathrm{JF}, \mathrm{PC}, \mathrm{UB}_{2}, \mathrm{ES}, \mathrm{SP}, \mathrm{DF}$

$\mathrm{BH}_{1}, \mathrm{JF}, \mathrm{ES}, \mathrm{SP}$

JF, SP

$\mathrm{BH}_{1}, \mathrm{BH}_{2}, \mathrm{JF}, \mathrm{MG}_{1}, \mathrm{PC}, \mathrm{UB}_{2}, \mathrm{ES}, \mathrm{SP}, \mathrm{DF}$

$\mathrm{BH}_{1}, \mathrm{BH}_{2}, \mathrm{JF}, \mathrm{PC}, \mathrm{UB}_{2}, \mathrm{ES}, \mathrm{SP}, \mathrm{DF}$

$\mathrm{BH}_{1}, \mathrm{JF}, \mathrm{ES}, \mathrm{SP}, \mathrm{DF}$

$\mathrm{BH}_{1}, \mathrm{BH}_{2}, \mathrm{JF}, \mathrm{PC}, \mathrm{ES}, \mathrm{SP}, \mathrm{DF}$

$\mathrm{BH}_{1}, \mathrm{PC}, \mathrm{ES}, \mathrm{SP}, \mathrm{DF}$

$\mathrm{BH}_{1}, \mathrm{JF}, \mathrm{PC}, \mathrm{ES}, \mathrm{SP}, \mathrm{DF}$

ES

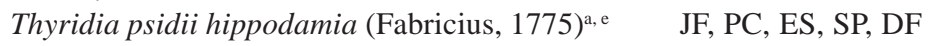

\begin{tabular}{|c|c|c|}
\hline Nymphalidae/Limenitidinae & $\begin{array}{l}\text { Adelpha lycorias }(\text { Godart, [1824]) } \\
\text { Adelpha syma (Godart, [1824]) })^{\mathrm{b}}\end{array}$ & $\begin{array}{l}\mathrm{PC}, \mathrm{ES}, \mathrm{SP} \\
\mathrm{BH}_{1}, \mathrm{PC}, \mathrm{ES}, \mathrm{SP}\end{array}$ \\
\hline Nymphalidae/Nymphalinae & $\begin{array}{l}\text { Anartia amathea (Linnaeus, 1758) } \\
\text { Eresia lansdorfi }(\text { Godart, } 1819)^{\mathrm{b}, \mathrm{e}} \\
\text { Hypanartia lethe (Fabricius, 1793) } \\
\text { Siproeta stelenes (Linnaeus, 1758) } \\
\text { Tegosa claudina }(\text { Eschscholtz, } 1821)^{\mathrm{b}, \mathrm{t}, \mathrm{e}}\end{array}$ & $\begin{array}{l}\mathrm{BH}_{1}, \mathrm{PC}, \mathrm{UB}_{2}, \mathrm{ES}, \mathrm{SP}, \mathrm{DF} \\
\mathrm{BH}_{1}, \mathrm{BH}_{2}, \mathrm{UB}_{2}, \mathrm{ES}, \mathrm{SP}, \mathrm{DF} \\
\mathrm{BH}_{1}, \mathrm{PC}, \mathrm{ES}, \mathrm{SP}, \mathrm{DF} \\
\mathrm{BH}_{1}, \mathrm{BH}_{2}, \mathrm{PC}, \mathrm{ES}, \mathrm{SP}, \mathrm{DF} \\
\mathrm{BH}_{2}, \mathrm{UB}_{2}, \mathrm{ES}, \mathrm{SP}, \mathrm{DF}\end{array}$ \\
\hline Nymphalidae/ Satyrinae & Hermeuptychia hermes (Fabricius, 1775) ${ }^{\mathrm{b}}$ & $\mathrm{BH}_{1}, \mathrm{BH}_{2}, \mathrm{PC}, \mathrm{UB}_{1}, \mathrm{UB}_{2}, \mathrm{ES}, \mathrm{SP}, \mathrm{DF}$ \\
\hline Papilionidae/ Papilioninae & $\begin{array}{l}\text { Battus polystictus }(\text { Butler, 1874) } \\
\text { Heraclides androgeus }(\text { Cramer, 1975) })^{2, \mathrm{e}} \\
\text { Heraclides astyalus (Godart, 1819) }{ }^{\mathrm{e}} \\
\text { Heraclides hectorides (Esper, 1794) }{ }^{\mathrm{b}, \mathrm{e}} \\
\text { Mimoides lysithous }(\text { Hübner, [1821]) } \\
\text { Parides anchises nephalion }(\text { Godart, 1819) }\end{array}$ & $\begin{array}{l}\mathrm{PC}, \mathrm{ES}, \mathrm{SP} \\
\mathrm{ES} \\
\mathrm{BH}_{1}, \mathrm{PC}, \mathrm{UB}_{2}, \mathrm{ES}, \mathrm{SP}, \mathrm{DF} \\
\mathrm{BH}_{1}, \mathrm{PC}, \mathrm{ES}, \mathrm{SP}, \mathrm{DF} \\
\mathrm{PC}, \mathrm{SP} \\
\mathrm{BH}_{1}, \mathrm{BH}_{2}, \mathrm{ES}, \mathrm{SP}, \mathrm{DF}\end{array}$ \\
\hline Pieridae/Coliadinae & $\begin{array}{l}\text { Eurema albula }(\text { Cramer, } 1776)^{\mathrm{b}, \mathrm{e}, \mathrm{t}} \\
\text { Eurema arbela } \text { Geyer, } 1832^{\mathrm{b}} \\
\text { Eurema } \mathrm{sp} . \text { Hübner, [1819] }]^{\mathrm{e}}\end{array}$ & $\begin{array}{l}\mathrm{BH}_{1}, \mathrm{PC}, \mathrm{UB}_{2}, \mathrm{ES}, \mathrm{SP}, \mathrm{DF} \\
\mathrm{BH}_{1}, \mathrm{ES}, \mathrm{SP}, \mathrm{DF} \\
-\end{array}$ \\
\hline Pieridae/ Dismorphiinae & $\begin{array}{l}\text { Dismorphia amphione astynome (Dalman, 1823) })^{2, \mathrm{e}} \\
\text { Dismorphia astyocha Hübner, [1831] }{ }^{\mathrm{b}} \\
\text { Dismorphia thermesia (Godart, 1819) } \\
\text { Enantia lina (Herbst, 1972), e } \\
\text { Pseudopieris nehemia (Boisduval, 1836) }\end{array}$ & $\begin{array}{l}\mathrm{ES}, \mathrm{SP}, \mathrm{DF} \\
\mathrm{BH}_{1}, \mathrm{BH}_{2}, \mathrm{PC}, \mathrm{ES}, \mathrm{SP} \\
\mathrm{BH}_{1}, \mathrm{PC}, \mathrm{ES}, \mathrm{SP}, \mathrm{DF} \\
\mathrm{ES}, \mathrm{SP}, \mathrm{DF} \\
\mathrm{BH}_{1}, \mathrm{BH}_{2}, \mathrm{PC}, \mathrm{ES}, \mathrm{DF}\end{array}$ \\
\hline Pieridae/ Pierinae & $\begin{array}{l}\text { Archonias brassolis tereas (Godart, 1819) } \\
\text { Hesperocharis anguitia (Godart, 1819) }\end{array}$ & $\begin{array}{l}\mathrm{BH}_{1}, \mathrm{MG}_{1}, \mathrm{PC}, \mathrm{UB}_{2}, \mathrm{ES}, \mathrm{SP}, \mathrm{DF} \\
\mathrm{BH}_{1}, \mathrm{MG}_{1}, \mathrm{PC}, \mathrm{ES}, \mathrm{SP}, \mathrm{DF}\end{array}$ \\
\hline Riodinidae/ Riodininae & $\begin{array}{l}\text { Charis sp. Hübner, [1819] }]^{\mathrm{e}} \\
\text { Emesis ocypore }(\text { Geyer, 1837) } \\
\text { Emesis sp. Fabricius, } 1807^{\mathrm{e}} \\
\text { Lemonias zygia Hübner, [1807] }]^{\mathrm{e}} \\
\text { Melanis alena }(\text { Hewitson, 1870) })^{2, \mathrm{e}} \\
\text { Nymphidium lisimon }(\text { Stoll, 1790) } \\
\text { Synargis phlisus phliasus }(\text { Clerck, 1764) } \\
\text { Thisbe irenea }(\text { Stoll, } 1780)^{2, \mathrm{e}}\end{array}$ & $\begin{array}{l}- \\
\mathrm{BH}_{1}, \mathrm{PC}, \mathrm{ES}, \mathrm{SP} \\
- \\
\mathrm{BH}_{1}, \mathrm{ES}, \mathrm{SP} \\
\text { Restricted to MP } \\
\mathrm{UB}_{2}, \mathrm{ES}, \mathrm{DF} \\
\mathrm{BH}_{1}, \mathrm{MG}_{2}, \mathrm{PC}, \mathrm{UB}_{2}, \mathrm{SP} \\
\text { Restricted to MP }\end{array}$ \\
\hline
\end{tabular}

${ }^{1}$ For abbreviations see Table 2.

${ }^{2}$ First record in Minas Gerais. 
Table 2. Lepidoptera inventories conducted in Distrito Federal, Espírito Santo, Minas Gerais and São Paulo: vegetation of study sites and number of collected species

\begin{tabular}{|c|c|c|c|}
\hline \multicolumn{2}{|c|}{$\begin{array}{l}\text { REGION } \\
\text { State/Municipality (Abreviation) }\end{array}$} & \multirow[t]{2}{*}{ Species(no.) } & \multirow[t]{2}{*}{ Source } \\
\hline MIDWEST & & & \\
\hline $\begin{array}{l}\text { Distrito Federal/ } \\
\text { Brasília (DF) }\end{array}$ & Cerrado & 839 & $\begin{array}{l}\text { Emery et al. (2006), Mielke } \\
\text { et al. (2008) }\end{array}$ \\
\hline \multicolumn{4}{|l|}{$\begin{array}{l}\text { SOUTHEAST } \\
\end{array}$} \\
\hline \multicolumn{4}{|l|}{ Espírito Santo/ } \\
\hline \multicolumn{4}{|l|}{ Minas Gerais/ } \\
\hline Poços de Caldas (PC) & Atlantic Forest / Seasonal Semideciduous Forest & 572 & Ebert (1969) \\
\hline Belo Horizonte $\left(\mathrm{BH}_{1}\right)$ & $\begin{array}{l}\text { Atlantic Forest / Seasonal Semideciduous Forest; } \\
\text { Cerrado; Rocky Fields; Urban Area }\end{array}$ & 269 & Brown Jr. \& Mielke (1968) \\
\hline Uberlândia $\left(\mathrm{UB}_{2}\right)$ & $\begin{array}{l}\text { Atlantic Forest / Seasonal Semideciduous Forest; } \\
\text { Cerrado; Urban Area }\end{array}$ & 251 & Motta (2002) \\
\hline Viçosa $(\mathrm{MP})^{1}$ & Atlantic Forest / Seasonal Semideciduous Forest & 233 & $\begin{array}{l}\text { Ferreira et al. }(1986 ; 1995) \text {, } \\
\text { Present study }\end{array}$ \\
\hline Belo Horizonte $\left(\mathrm{BH}_{2}\right)$ & Atlantic Forest / Seasonal Semideciduous Forest & 91 & Silva et al. (2007) \\
\hline$?\left(\mathrm{MG}_{2}\right)$ & $?$ & 45 & Stichel (1923) \\
\hline Uberlândia $\left(\mathrm{UB}_{1}\right)$ & Urban Area, Gallery Forest & 36 & Fortunato \& Ruszczyk (1997) \\
\hline$?\left(\mathrm{MG}_{1}\right)$ & $?$ & $\simeq 31$ & May (1924) \\
\hline Juiz de Fora $(\mathrm{JF})^{2}$ & Atlantic Forest / Seasonal Semideciduous Forest & 18 & Costa (2002) \\
\hline \multicolumn{4}{|l|}{ São Paulo/ } \\
\hline $\begin{array}{l}\text { Cabreúva, Cajamar, } \\
\text { Jundiaí and Pirapora } \\
\text { do Bom Jesus (SP) }\end{array}$ & Atlantic Forest / Seasonal Semideciduous Forest & 652 & Brown Jr. (1992) \\
\hline
\end{tabular}

Table 3. Families, subfamilies and number of butterfly species (Lepidoptera: Rhopalocera) in common between the Seasonal Semideciduous Forest in the forest reserve Mata do Paraíso, Viçosa (MP) and other areas in Minas Gerais, São Paulo, Espírito Santo and Distrito Federal (for abbreviations, see Table 2)

\begin{tabular}{|c|c|c|c|c|c|c|c|c|c|c|c|c|}
\hline \multirow{3}{*}{ Families } & \multirow{3}{*}{ Subfamilies } & \multicolumn{11}{|c|}{ Collection sites } \\
\hline & & \multicolumn{8}{|c|}{ Minas Gerais } & \multirow{2}{*}{$\frac{\begin{array}{c}\text { São } \\
\text { Paulo }\end{array}}{\text { SP }}$} & \multirow{2}{*}{$\begin{array}{c}\text { Espírito } \\
\text { Santo } \\
\text { ES }\end{array}$} & \multirow{2}{*}{$\begin{array}{l}\text { Distrito } \\
\text { Federal } \\
\text { DF }\end{array}$} \\
\hline & & MP & $\mathbf{P C}$ & $\mathrm{BH}_{1}$ & $\mathbf{U B}_{2}$ & $\mathbf{B H}_{2}$ & $\mathbf{M G}_{1}$ & $\mathrm{UB}_{1}$ & $\mathbf{M G}_{2}$ & & & \\
\hline \multirow[t]{2}{*}{ Hesperiidae } & Hesperiinae & 6 & 6 & 2 & 3 & - & - & - & - & 6 & 5 & 4 \\
\hline & Pyrginae & 19 & 13 & 11 & 8 & 7 & - & - & - & 19 & 9 & 17 \\
\hline \multirow[t]{9}{*}{ Nymphalidae } & Acreinae & 4 & 2 & 2 & 2 & 2 & - & 1 & - & 4 & 3 & 3 \\
\hline & Biblidinae & 2 & 2 & 2 & 2 & 1 & 2 & 1 & - & 2 & 2 & 1 \\
\hline & Cyrestinae & 1 & 1 & 1 & - & - & - & - & - & 1 & 1 & 1 \\
\hline & Danainae & 1 & - & - & - & 1 & - & - & - & 1 & 1 & 1 \\
\hline & Heliconiinae & 9 & 6 & 8 & 5 & 3 & - & - & - & 9 & 9 & 7 \\
\hline & Ithomiinae & 23 & 12 & 13 & 8 & 5 & - & - & - & 21 & 19 & 14 \\
\hline & Limenitidinae & 2 & 2 & 1 & - & - & - & - & - & 2 & 2 & - \\
\hline & Nymphalinae & 5 & 3 & 4 & 3 & 3 & - & - & - & 5 & 5 & 5 \\
\hline & Satyrinae & 1 & 1 & 1 & 1 & 1 & - & 1 & - & 1 & 1 & 1 \\
\hline Papilionidae & Papilioninae & 6 & 4 & 3 & 1 & 1 & - & - & - & 5 & 5 & 3 \\
\hline \multirow[t]{3}{*}{ Pieridae } & Coliadinae & 2 & 1 & 2 & 1 & - & - & - & - & 2 & 2 & 2 \\
\hline & Dismorphiinae & 5 & 3 & 3 & - & 2 & - & - & - & 4 & 5 & 4 \\
\hline & Pierinae & 2 & 2 & 2 & 1 & - & 2 & - & - & 2 & 2 & 2 \\
\hline Riodinidae & Riodininae & 6 & 2 & 3 & 2 & - & - & - & 1 & 3 & 3 & 1 \\
\hline \multicolumn{2}{|c|}{$\begin{array}{l}\text { Number and percentage of } \\
\text { species in common with the MP }\end{array}$} & 94 & $\begin{array}{c}60 \\
(63.82)\end{array}$ & $\begin{array}{c}58 \\
(61.70)\end{array}$ & $\begin{array}{c}37 \\
(39.36)\end{array}$ & $\begin{array}{c}26 \\
(27.66)\end{array}$ & $\begin{array}{c}4 \\
(4.25)\end{array}$ & $\begin{array}{c}3 \\
(3.19)\end{array}$ & $\begin{array}{c}1 \\
(1.06)\end{array}$ & $\begin{array}{c}87 \\
(92.55)\end{array}$ & $\begin{array}{c}74 \\
(78.72)\end{array}$ & $\begin{array}{c}66 \\
(70.21)\end{array}$ \\
\hline
\end{tabular}

Rev. Ceres, Viçosa, v. 59, n.5, p. 571-579, set/out, 2012 


\section{DISCUSSION}

The results presented in this study showed that plants (Asteraceae species) can be important tools for monitoring anthophilous insects, as suggested by Frankie et al. (2002). These authors monitored bee species on Bignoniaceae flowers in tropical areas of Costa Rica and California. Results confirming the usefulness of plants for monitoring anthophilous butterflies were published by Fonseca et al. (2006). These authors recorded 98 butterfly species on flowers of Stachytarpheta cayennensis (Rich.) Vahl. (Verbenaceae) and concluded that flowers of this species are critical for maintaining the community of Lepidoptera visitors. In this study, we focused on the species A. brasilianum and $E$. intermedium, belonging to genera regularly visited by Lepidoptera (Brown Jr., 1984a; 1987; 1992).

The richness of anthophilous butterflies in Mata do Paraíso may reflect the richness of the flora of the reserve, creating conditions for the survival of these insects. For butterflies in the adult stage, nectar is provided by psychophilous flowers, and other plants provide leaves for the larvae. For example, larvae of Nymphalidae, the most representative group of butterflies found in Mata do Paraíso, feed on leaves of Solanaceae, Apocynaceae and Passifloraceae (Brown Jr., 1992), families that are wellrepresented in the studied site (according to the collection of the VIC Herbarium).

The largest number of the butterfly species Nymphalidae and Hesperiidae confirmed their status as important pollinator group (Brown Jr., 1992; Fonseca et al., 2006; Godinho, 2007; Lelis, 2008; Cruz, 2009; Fonseca, 2009). The high number of Ithomiinae species may be due to the richness of Eupatorium (at least 11 species), in addition to the occurrence of $A$. brasilianum. Ithomiinae evolved the ability to assimilate pyrrolizidine alkaloids (PAs) from the nectar of these Asteraceae and transform them into compounds required to complete their life cycle (Brown Jr., 1984a; b; 1987; Trigo et al., 1996; Paiva \& Barata, 2004). This illustrates the importance of Asteraceae plants in maintaining butterfly populations in a given vegetation formation.

The low representation of anthophilous butterflies of the families Papilionidae and Pieridae was also observed in the study of Fonseca et al. (2006). These authors reported 11 species of Pieridae and four of Papilionidae and a large number of Hesperiidae (72 species) visiting flowers of $S$. cayennensis. This similarity with our results is probably due to characteristics the two study sites have in common (semideciduous forest). However, Fonseca et al. (2006) recorded only seven species of Nymphalidae, which may be related with the absence of PAs required by Ithomiinae in the nectar of $S$. cayennensis.

In our study, the few species of Riodinidae that were observed confirmed the study of Callaghan (1978), i.e., populations of these butterflies are characterized by frequency in low intensity.

Records of Thyridia psidii cetoides [= Thyridia psidii hippodamia (Fabricius)], very rare in disturbed forest (Brown Jr., 1992), indicate that Mata do Paraíso contains well-preserved habitats. The existence of this species at a given site may be related to the topography and preserved vegetation that support the persistence of relict populations, very rare or with few known populations (Brown Jr. \& Freitas, 2000). These results show that in Mata do Paraíso, conditions are given for maintaining a high diversity of butterfly species, including some of the most demanding in terms of habitat conditions. In contrast, some subfamilies reported to be favoured by forest fragmentation by Uehara-Prado et al. (2003) were poorly registered in Mata do Paraíso (for example Biblidinae, represented by Biblis hyperia nectanabis (Fruhstorfer, 1909) and Temenis laothoe meridionalis Ebert, 1965).

The almost complete similarity between the butterfly species of Mata do Paraíso and São Paulo was expected. In the latter region, the survey was carried out in an area of 10,000 ha with mainly the same semideciduous forest vegetation as in former one (Brown Jr., 1992; Leitão-Filho, 1992), a factor that raises the chances of recording species in common. These results and other studies reporting high similarities showed that more representative inventories of the butterfly fauna of a given site increase the chances of recording species in common, regardless of the type of vegetation.

This latter finding shows that the habitat of many butterflies comprises forests and open and dry areas, such as Cerrado. According to Brown Jr. \& Freitas (2000), species of Papilionidae, Pieridae and Nymphalidae observed in Mata do Paraíso occur in Cerrado, Atlantic Forest, on the coast of Bahia, in inland mountainous areas and especially in the coastal plain of "Baixada Fluminense", Rio de Janeiro, Rio de Janeiro State. The capacity of these butterflies to explore different habitats and, consequently, different abiotic conditions resulted in their wide geographic distribution.

The richness of Lepidoptera in Mata do Paraíso was certainly underestimated, since frugivorous butterflies and diurnal and nocturnal moths were not included, except Sphingidae and Arctiidae (Ferreira et al., 1986; 1995). The 15 new records increase the richness of the lepidopteran fauna of Minas Gerais and reveal the lack of systematic inventories to represent its diversity. The finding of two butterflies only in Mata do Paraíso (M. alena and T. irenea) indicates an apparently greater environmental restriction of these insects or their low frequency (Callaghan, 1978), which may hamper sampling by the low number of individuals, as they are not protected as rare or endangered species (Casagrande et al., 1998; Brown Jr. \& Freitas, 2000; Machado et al., 2008). 
The proportions of species in families and subfamilies of butterflies in tropical forests of Central and South America are relatively constant (Beccaloni \& Gaston, 1995). These authors argued that there is a strong positive correlation between species richness of Ithomiine and the overall species richness of all other butterflies across all areas, and the proportion of Ithomiinae (as an indicator group) is reasonably invariant. They concluded that it should be possible to use the mean proportion (4.6\%) to predict the overall butterfly species richness of an area for which the total number of Ithomiine is known.

Based on these statements and on the identification of all Ithomiinae in Mata do Paraíso (23 species) by the current inventory, the richness of this fragment would be 500 butterfly species distributed in different families and subfamilies. This estimate, if confirmed, highlights the relevance of this fragment in maintaining the lepidopteran population and indicates the need of further similar work to understand the richness of its Lepidoptera fauna.

In this paper, we present the first inventory of anthophilous butterflies of a semideciduous forest fragment in the Zona da Mata, Minas Gerais State. It includes 15 new records for the State, two of which restricted to the study site, and it shows the importance of forest fragments, particularly of the forest reserve Mata do Paraíso, as a last refuge for these insects. The survival of butterflies depends on the conservation of plants, as nectar source for the adults and leaves for the larvae. It is noteworthy that the mutualistic interactions between butterflies and psychophilous flowers, including those in Mata do Paraíso, call for further investigation.

\section{CONCLUSIONS}

Asteraceae species, especially E. intermedium, $B$. segetum and A. brasilianum, are important tools for monitoring anthophilous butterflies.

In these plants, 108 butterflies species were collected, belonging to 70 genera, 16 subfamilies and five families, representing the fourth largest lepidopteran survey of Minas Gerais State.

There is high similarity (above 60\%) among the species of butterflies anthophilous of Mata do Paraíso and of the lepidopteran inventories made in Southeastern and Midwestern regions of Brazil, regardless of the type of vegetation.

\section{ACKNOWLEDGEMENTS}

We thank the taxonomists Dr. Keith S. Brown Jr. (Unicamp), Dr. André V. L. Freitas (Unicamp) and Ronaldo Bastos Francini (Unisantos) for their help with species identification and CNPq for the financial support, and the research fellowship for the last author (Grant no. 473609/ 2007-0). This work is part of the master's theses of the first four authors (Graduate Program in Botany at UFV).

\section{REFERENCES}

Beccaloni GW \& Gaston KJ (1995) Predicting species richness of Neotropical forest butterflies: Ithomiinae (Lepidoptera: Nymphalidae) as indicators. Biological Conservation, 71:77-86.

Brown Jr. KS (1984a) Adult-obtained pyrrolizidine alkaloids defend ithomiinae butterflies against predator. Nature, 309:707-709.

Brown Jr. KS (1984b) Chemical ecology of dehydropyrrolizidine alkaloids in adult Ithomiinae (Lepidoptera: Nymphalidae). Revista Brasileira de Biologia, 44:435-446.

Brown Jr. KS (1987) Chemistry of the Solanaceae/Ithomiinae interface. Annals of the Missouri Botanical Garden, 74:359-422.

Brown Jr. KS (1992) Borboletas da Serra do Japi: diversidade, hábitats, recursos alimentares e variação temporal. In: Morellato LPC (Ed.) História natural da Serra do Japi: ecologia e preservação de uma área florestal no Sudeste do Brasil. Campinas, Unicamp. p.142-187.

Brown Jr. KS \& Freitas AVL (2000) Diversidade de Lepidoptera em Santa Teresa, Espírito Santo. Boletim do Museu de Biologia Mello Leitão (N. Sér.), 11/12:71-116.

Brown Jr. KS \& Mielke OHH (1968) Lepidoptera of the Central Brazil plateau. III. Partial list for the Belo Horizonte area, showing the character of the southeastern "blend zone". Journal of the Lepidopterists' Society, 22:147-157.

Callaghan CJ (1978) Studies on restinga butterflies. II. Notes on the population structure of Menander felsina (Riodinidae). Journal of the Lepidopterists' Society, 32:37-48.

Casagrande MM, Mielke OHH \& Brown Jr KS (1998) Borboletas (Lepidoptera) ameaçadas de extinção em Minas Gerais, Brasil. Revista Brasileira de Zoologia, 15:241-259.

Costa FAPL (2002) Borboletas Ithomiinae (Lepidoptera, Nymphalidae) da Reserva Biológicas D’Anta (Juiz de Fora, MG). Revista Brasileira de Zoologia, 4:143-149.

Cruz KC (2009) Biologia reprodutiva e polinizadores de Eupatorium intermedium DC. (Asteraceae) em fragmento de Floresta Atlântica. Dissertação de Mestrado. Universidade Federal de Viçosa, Viçosa. 66p.

Departamento Nacional de Meteorologia (1992) Normais climatológicas (1961-1990). Brasília, SPI/EMBRAPA. 84p.

Ebert H (1969) On the frequency of butterflies in eastern Brazil, with a list of butterfly fauna of Poços de Caldas, Minas Gerais. Journal of the Lepidopterists' Society, 23:1-48.

Emery EO, Brown Jr KS \& Pinheiro CEG (2006) As borboletas (Lepidoptera, Papilionoidea) do Distrito Federal, Brasil. Revista Brasileira de Entomologia, 50:85-92.

Ferreira PSF, Martins DS \& Hubner N (1986) Inventário, flutuação e análise entomofaunística em mata remanescente da Zona da Mata, Viçosa, Minas Gerais. I. Sphingidae: Lepidoptera. Revista Ceres, 33:516-527.

Ferreira PSF, Paula AS \& Martins DS (1995) Análise faunística de Lepidoptera Arctiidae em área de reserva natural remanescente de floresta tropical em Viçosa, Minas Gerais. Anais da Sociedade Entomológica do Brasil, 24:123-133.

Fonseca NG, Fumagai AF \& Mielke OHH (2006) Lepidópteros visitantes florais de Stachytarpheta cayennensis (Rich.) Vahl (Verbenaceae) em remanescente de Mata Atlântica, Minas Gerais, Brasil. Revista Brasileira de Entomologia, 50:399-405. 
Fonseca RS (2009) Biologia reprodutiva de Tilesia baccata (L.) Pruski (Heliantheae, Asteraceae): fenologia, polinização e dispersão. Dissertação de Mestrado. Universidade Federal de Viçosa, Viçosa. 80p

Fortunato L \& Ruszczyk A (1997) Comunidades de lepidópteros frugívoros em áreas verdes urbanas e extraurbanas de Uberlândia, MG. Revista Brasileira de Biologia, 57:79-87.

Frankie WG, Vinson SB, Thorp RW, Rizzardi MA, Tomkins MM \& Newstrom-Loyd LE (2002) Monitoring, an essential tool in bee ecology and conservation. In: Kevan PG \& ImperatrizFonseca VL (Eds.) Pollinating bees - The conservation link between agriculture and nature. Brasília, Ministry of Environment. p.187-198.

Fundação SOS Mata Atlantica (2009) Atlas remanescentes florestais da Mata Atlântica 2005-2008 (Relatório parcial). São Paulo, Fundação SOS Mata Atlântica \& INPE. 156p.

Godinho MAS (2007) Biologia reprodutiva e germinação de sementes em Adenostemma brasilianum (Pers.) Cass. (Asteraceae). Dissertação de Mestrado. Universidade Federal de Viçosa, Viçosa. 77p.

IEF (2010) Cobertura vegetal de Minas Gerais. Disponível em: <http://www.ief.mg.gov.br/florestas >. Acessado em: 20 de junho de 2010

Leitão-Filho HF (1992) A flora arbórea da Serra do Japi. In: Morellato LPC (Ed.) História natural da Serra do Japi: ecologia e preservação de uma área florestal no Sudeste do Brasil. Campinas, Unicamp. p.40-62

Lelis SM (2008) Biologia reprodutiva de Bidens segetum Mart. ex Colla (Heliantheae, Asteraceae). Dissertação de Mestrado. Universidade Federal de Viçosa, Viçosa. 45p.

Machado ABM, Drummond GM \& Paglia AP (2008) Livro vermelho da fauna brasileira ameaçada de extinção. Brasília, MMA \& Fundação Biodiversitas. 510p.

Mani MS \& Saravanan JM (1999) Pollination ecology and evolution in Compositae (Asteraceae). New Hampshire, Science Publishers, Inc. 166p.
May E (1924) Relatório das excursões effectuadas nos estados de Rio, Minas Geraes e Bahia. Boletim do Museu Nacional, 1:367375 .

Mielke OHH, Emery EO \& Pinheiro CEG (2008) As borboletas Hesperiidae (Lepidoptera, Hesperioidea) do Distrito Federal, Brasil. Revista Brasileira de Entomologia, 52:283-288.

Motta PC (2002) Butterflies from the Uberlândia region, central Brazil: species list end biological comments. Brazilian Journal of Biology, 62:151-163.

Paiva JA \& Barata LES (2004) Pirrolizidine alkaloids in three Senecio species from Southern Brazil. Biochemical Systematics and Ecology, 32:1219-1222.

Pinto SIC, Martins SV, Silva AG, Scoss LM \& Dias HCT (2007) Estrutura do componente arbustivo-arbóreo em dois estádios sucessionais de floresta estacional semidecidual na Reserva Florestal Mata do Paraíso, Viçosa, MG, Brasil. Revista Árvore, 31:823-833.

Silva ARM, Landa GG \& Vitalino RF (2007) Borboletas (Lepidoptera) de um fragmento de mata urbano em Minas Gerais, Brasil. Lundiana, 8:137-142.

Stichel H (1923) Beiträge zur Kenntnis der Riodinidenfauna Südamerikas. III. Minas Gerais. Zeitschrift für wissenschaftliche Insektenbiologie, 18:1-9

Trigo JR, Brown Jr KS, Henriques AS \& Barata LES (1996) Qualitative patterns of pyrrolizidine alkaloids in Ithomiinae butterflies. Biochemical Systematics and Ecology, 24:181-188.

Uehara-Prado M, Freitas AVL, Metzger JP, Alves L, Silva WG \& Brown Jr KS (2003) Borboletas frugívoras (Lepidoptera: Nymphalidae) como indicadoras de fragmentação florestal no Planalto Atlântico Paulista. In: $7^{\circ}$ Congresso de Ecologia do Brasil, Fortaleza. Anais de Trabalhos Completos do VI Congresso de Ecologia do Brasil, Editora da Universidade Federal do Ceará. p.297-299.

Veloso HP, Rangel-Filho AL \& Lima JCA (1991) Classificação da vegetação brasileira, adaptada a um sistema universal. Rio de Janeiro, Fundação Instituto Brasileiro de Geografia e Estatística. 584p. 